\title{
artículos
}

\section{La indización por materia de las monografías en los catálogos en línea de bibliotecas generales}

\author{
Ana María Martínez \\ Licen cia daen Bibliote co logía y Do cu men ta ción y profe sora \\ titular. Departamento de Bibliotecología de la Facultad de \\ Hu mani da des y Cien cias de la Edu ca ción, Uni ver sidad Na \\ cio nal de La Pla ta, 48 y 6-5 $5^{\circ}$ piso, $1900 \mathrm{La} \mathrm{Pla} \mathrm{ta,} \mathrm{Ar} \mathrm{gen} \mathrm{ti} \mathrm{na}$
}

\author{
Mariana Pichinini \\ Biblio te caria Do cu men talis ta, ads cri ta a la Cá te dra de Cla si \\ ficación I. Departamen to de Biblio te co logía de la Facultad de \\ Hu ma ni da des y Cien cias de la Edu ca ción, Uni ver si dad Na \\ cio nal de La Pla ta, 48 y 6-5 ${ }^{\circ}$ piso, $1900 \mathrm{La} \mathrm{Pla} \mathrm{ta,} \mathrm{Ar} \mathrm{gen} \mathrm{ti} \mathrm{na}$
}

\begin{abstract}
RESUMEN
$\boldsymbol{E}$ nesta re vi sión se re se ñan al gu nos proble mas re la cio na dos con la in di za ción por ma te ria de las mo no gra fías en los ca tálogosen línea de bi blio te cas ge ne ra les, que pue den re su mirse en la esca sa can ti dad de tér mi nos des crip ti vos en cada re gis tro bi bliográfico, las incoherencias en la indización y las limitaciones de los lenguajes de indización. Se han propuesto tres solu cio nes: au men tar los pun tos de ac ce so por ma te ria en cada re gis tro, com bi nan do las pa la bras cla ves de la len gua na tu ral con tér mi nos verba les de in di za ción (en cabe za mien tos de ma te ria y descrip tores) ysistemáticos (notaciones); facilitarelacce so en lí nea al ca tá lo go de au to ri da des y al len gua je de in di za ción tan to para losin di za do res como para los usua rios; y me jorar los len gua jes de in di za ción. Esta úl ti ma op ción se ha vis to afec ta da por las di fi cul ta des eco nó mi cas que im pi den o ha cen muy di fícil la crea ción de nue vos len gua jes de in di za ción o la re es truc tu ra ción de aque llos tra di cio na les como las lis tas de encabe za mien tos de ma teria y las cla si fi ca ciones de ci ma les, y más aún, la re cla sifica ción o re in di za ción de las co lec cio nes existentes. Las experiencias obtenidas en los países desarrollados pueden ayudar a los bibliotecarios latinoamericanos a discutirestos proble mas, prevenir los mismos errores y brindar mejores ca tá lo gos en línea a los usua rios.
\end{abstract}

\section{Abstract}

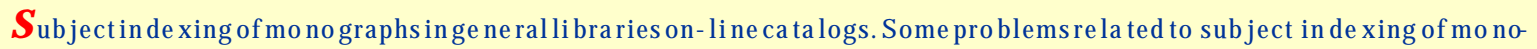
graphs in ge ne ral li bra ries on- li ne ca ta logs are re viewed. The se problems in clu de thesmall num ber of des crip ti ve terms in bibliographical records, inconsistency of indexing, and obsolescence of indexing languages. Three solutions have been pro po sed: to in crea se the sub ject ac cess points for each bi blio gra phi cal re cord, com bining keywords from na tu ral lan gua ge (tit le and abs tract or con tent note) with ver bal con tro lled terms (sub ject hea dings or descriptores), and notationalcon trolled terms (clas sifica tions); to provide on-li ne ac cess to authority fi les and in de xing langua ges, both for in de xers and users, and to im pro ve the in de xing lan gua ges. This last pro po sal has been affected by eco no mi cal issues, ma king it very diffi cult to crea te or rea rran ge tho se tra di tio nal in de xing lan gua ges like sub ject hea dings lists and de ci mal clas si fi ca tions, as well as the re classification or re in de xing of li brary collec tions. The ex pe rien ces ob tai ned in de velo ped coun tries may help La tin Ame ri can librarians to discuss these problems, to pre vent the same errors, and to offer bet ter on-li ne catalogs for users.

$\mathrm{E}$ 1 acceso por materia a la información E es la característica que distingue al catálogo de biblioteca de un simple inventario y que lo convierte en un instrumento de recuperaciónde información y no sólo de datos.

Sin em bar go, a pe sar de los ade lan tos alcanzados por la tecnología, aún no se ha podido dar a los usuarios una respuesta satisfactoria en este aspecto, sobre todo en lo que concierne a la indización por materia de las monografías en los catálogos en línea de bibliotecas generales.

Inicialmente, alcomputarizarse los catálogos, la decisión de los bibliotecarios fue muy sencilla, inclu soinocente:pasar la fi cha de $7.5 \times 12.5 \mathrm{~cm}$ a la má qui na, es decir, reciclar el catálogo tradicional en un ambiente electrónico (Bates, 1989; Culkin, 1989; Lancaster, 1991; Su,
1994). En opinión de Mary Dykstra (1989) mu chas de las ex pe rien cias ac tuales son sólo catálogos de fichas sobre ruedas, que han arrastrado consigo lo que Klugman (1989) y Larson (1991a) denominan problemas endémicos de la indización.

Paradójicamente, la búsqueda por materia es la más solicitada por los usuarios - $59 \%$ de to das las bús que das — y la que 
mayor índice de fracasos presenta $-40 \%$ de respuestas con cero registros recuperados (Kern-Simirenko, 1983; Larson, 1991b; Peters, 1989).

Este alto índice de fracasos ha sido relaciona do con la fal ta de coin ci den cia en tre el vocabu la rio del usua rio y el vocabu lario del sistema. Alyson Carlyle (1989), de la Uni ver sity of Ca li for nia at Los Angeles, ha calculado que el promedio de coin ci den cia en tre los tér mi nos del usuario y los encabezamientos de materia de la Library of Congress (LCSH) es de un $50 \%$, mientras que Cousins (1992) de Gran Bretaña, ob tu vo $62 \%$ de coin ci dencia para LCSH y $30 \%$ para la Clasificación De ci mal de Dewey (CDD) en una biblioteca politécnica y $73 \%$ y $36 \%$ respectivamente en una biblioteca pública.

Esta fal ta de coin ci den cia ha sido atri buida a tres cau sas prin ci pa les: po cos tér minos descriptivos en cada registro bibliográfico, in cohe ren cias en la in di za ción y limitaciones de los lenguajes de indización.

Comoso lu ción se ha pro pues to au men tar los pun tos de ac ce so por ma te ria, fa cilitar el ac ce so en línea al ca tálo go de au to ri dades y al len gua je de in di za ción y me jo rar estos últimos (Bates, 1989; Buckland, 1992; Larson, 1991b; Su, 1994).

\section{LOS PUNTOS DE ACCESO POR MATERIA}

El catálogo de una biblioteca general, lo mis mo en fi chas que en com pu ta do ra, incluye principalmente monografías. Por lo ge ne ral, la in di za ción por ma te ria se limi ta a un ni vel mo no grá fi co, sin re su men y con es ca sos tér mi nos de in di za ción. En los Es ta dos Uni dos se ha cal cu la do que el promedio de encabezamientos de materia en los catálogos en línea de bibliotecas generales es de 1.8 por registro, con lo cual los usuarios no pueden lograr ni una adecuada exploración de los términos ni mucho menos una combinación con los operadores booleanos.

En 1977 Pau li ne A. Cochra ne, de la Syra cuse University de Estados Unidos, propuso aumentar los puntos de acceso por materia para los registros de monografías, incluyendo los términos de sumarios e índices analíticos en el campo de resumen, para ser recuperados por lengua natural. Con el fin de evaluar esta propuesta, se comparó un catálogo de 1979 monografías en humanidades y cien cias so ciales, cuyain di za ción se hizo conformato MARC, por un lado, y con el formatoaumentado, por el otro; esta últi ma op ción se de no mi nó BOOKS y contaba con un promedio de 32.4 términos descriptivos de materia por registro. En 90 búsquedas de prueba la recuperación promedio con MARC fue de 56 registros en 8 minutos, mientras que con BOOKS fue de 130 registros en 4 minutos $-233 \%$ de los registros MARC en $50 \%$ del tiempo (Settel y Cochrane, 1982).

A pesar de ciertas consideraciones económicas (Holley y Killheffer, 1982), la idea de aumentar los puntos de acceso por ma te ria ha sido acep ta da y no se ha limi ta do al uso de la len gua na tu ral. Como es sabido, la combinación de la lengua natural con los lenguajes de indización mejora la calidad de la recuperación, ya que permite aumentar tanto la certeza (recall) como la pre ci sión, aun que de diferente manera (Aitchison y Gilchrist, 1987; Dubois, 1987; Lancaster, 1991; Svenonious, 1986):

a) La lengua natural aumenta la certeza cuando se incluyen resúmenes extensos y texto completo, por la exhaustivi dad de la co ber tu ra y au men ta la precisión por el nivel de especificidad y actualización de sus términos.

b) El lenguaje de indización aumenta la certeza mediante el control de sinónimos y las relaciones asociativas, mien tras que au men ta la pre ci sión por el uso de tér mi nos com pues tos, el control de homónimos y polisemas y la utilización de vínculos y roles.

Shirley A. Cousins (1992), del University College of Wales de Gran Bretaña, tradujo los términos de sumarios e índices analíticos a PRECIS. Realizó tres pruebascondistin tos resul tados: for mato MARC sin tér mi nos de su ma rios eín dices $13 \%$ de certeza; formato aumentado recu pe ra do por len gua na tu ral $76 \%$ de certeza; formatoaumentadorecu peradopor PRECIS $81 \%$ de cer te za. La di fe ren cia de certeza entre el formato MARC y el formato aumentado es concluyente; la diferencia entre la lengua natural y PRECIS pa re ce nojus ti fi car el cos to de la tra ducción de los términos de sumarios e índices a un lenguaje de indización, pero es innegable que logró mejorar la certeza.
En forma similar, Gunnar Knutson (1991) lle vó a cabo una in ves ti ga ción en la Uni ver sity of Illi nois at Chi ca go con el propósito de establecer la relación entre el formato aumentado y el uso de la colección, evaluado mediante la circulación. Dividió su colección experimental en tres gru pos: a) re gis troMARC au mentado con más encabezamientos de materia (promedio de 7.5 encabezamientos por registro) y una nota de contenido en len gua natural, b) re gis troMARC co mún, y c) registro MARC aumentado sólo con la nota de con te nido. Lacirculación para el pri mer gru po fue de $46 \%$, mien tras que para los otros dos fue de $26 \%$ y $28 \%$ respectivamente.

Tam bién se han au men ta do los pun tos de accesoutilizandoesquemas de clasificación como la Li brary of Con gress Clas sification (LCC), la Dewey Decimal Classification (CDD) y la Clasificación Decimal Universal (CDU).

Ray R. Larson (1991a), de la University of California at Berkeley, ha desarrollado el sis te ma Cheshi re que fun cio na de la siguiente manera: 1) los registros documentales o bibliográficos son copiados de un ser vi cio de pro ce sos téc ni cos;2) la signaturatopográfica(callnumber) asigna da por la Li brary of Con gress con LCC es reprocesada, eliminando la signatura librística(Cutternumber) y con servan do solamente la signatura de clase (class number), que de esta forma se convierte - automáticamente- en una notación de LCC ; 3) el sistema consta de dos tipos de registros: el registro bibliográfico conven cional con los da tos del documento y el registro recolector (cluster record), que agrupa los títulos y los encabezamientos de materia de todos los registros bibliográficos que poseen una idéntica notación de LCC. En el momento de la búsqueda, cuando el usuario ingresa sus términos en lengua natural, el sis te ma le pre sen ta en pan ta lla el re gis tro recolector; entonces el usuario selecciona el títu lo o el en cabe za mien to de ma teria y el sistema recupera y exhibe los registros bibliográficos correspondientes. Así, 30.471 registros bibliográficos que contenían 221.042 encabezamientos de materia fue ron re uni dos bajo 8.435 notaciones; Lar son opi na que esta mo da li dad permite suplir las relaciones equivalentes y asociativas de un tesauro. 


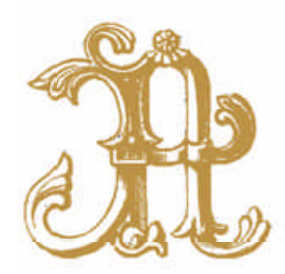

Durante mucho tiempo se ha postulado que los lenguajes precoordinados no son aptos para la computadora. Sin embargo, la Reunión Satélite de IFLA sobre Indización, realizada en Lisboa, Portugal puso de manifiesto que los sistemas precoordinados han sido preferidos para los catálogos en línea de las bibliotecas generales en numerosos países.

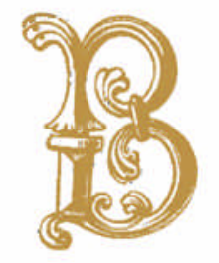

ElDewey DecimalClas sifica tion On line Project fue una investigación realizada en los años ochenta y financiada por el Council of Library Resources, OCLC y Forest Press, para evaluar la capacidad de CDD como instrumento de recuperación por materia en el catálogo en línea. Los resultados han sido informados en distintos trabajos (Drabenstott et al., 1990; Markey, 1987). El procedimiento propuesto en este caso es el siguiente:

1) el registro documental se copia de un servi cio de pro ce sos téc ni cos, incluyendo la notación de CDD;

2) la biblioteca posee una versión de CDD en línea con la notación y los términos verbales equivalentes (deno mi na ción de la cla se, tér mi nos del índice relativo, notas, etcétera);

3) el sis te ma hace coin ci dir la no ta ción del re gis tro do cu men tal con la no tación de CDD en línea y los términos verbales equivalentes de esta última se copian automáticamente en el registro bibliográfico.

La cantidad de términos verbales por registro aumentó en forma sustancial con un promedio de 19.6. La denominación de la cla se y los tér mi nos del ín di ce re lati vo deCDD han resul ta do tan ade cuados para la recuperación como LCSH y las palabras claves de título. Además, dado que CDD es un esquema jerárquico, se ofrece al usuario la opción de explorar clases más genéricas o más específicas. Mucho antes que las experiencias anteriores - en 1968-el American Ins ti tu te of Physics de Estados Unidos desarrolló un pro yec to de in ves ti ga ción so bre eluso de CDU (edi ción es pe cia li za da para ciencia y tec no lo gía nu clear) en sis te mas automatizados, incluyendo un catálogo en línea denominado AUDACIOUS.

En este catálogo, el usuario ingresa sus tér mi nos de bús que da en len gua na tu ral y el sis te ma le mues tra en pan ta lla to das las cla ses de CDU cu yas de no mi na cio nes incluyan dichos términos. Una vez que el usuario selecciona una clase, el sistema exhibe el esquema jerárquico de CDU, permitiendo la exploración de lo general a lo particular y viceversa (Buxton, 1990).

Un de sa rro llo si mi lar es el deETHICS de Zurich, Suiza. El sistema consta de un componente jerárquico de conceptos; a cada uno de estos conceptos le correspon de una no ta ción deCDU y nu me ro sos términos verbales, el principal de los cuales es considerado como un descriptor y el restocomosinónimos, formando entre todos un vocabulario controlado. En adición, los términos verbales se encuentran en tres idiomas (inglés, francés y ale mán). Cuan do el usua rio in gre sa sus términos de bús que da pue de op tar por la lengua natural o por el vocabulario controlado; luego el sistema le muestra en pantalla todas las clases de CDU cuyas denominaciones incluyan los términos ingresados, permitiéndole acceder a una exhibición sistemática en pantalla y explo rar las cla ses más ge né ri cas o más específicas. El usuario tam bién pue de solicitar su búsqueda por la notación, en cuyocasoel sis te ma exhibe directamen te el orden sistemático. ETHICS cuen ta con más de 50.000 cla ses deCDU y 1.3 mi llones de descriptores (Buxton, 1990).

Durante mucho tiempo se ha postulado queloslenguajes precoordinados ( LCSH, LCC, CDD, CDU) no son aptos para la computadora. Sin embargo, la Reunión Satélite de IFLA sobre Indización, realizada en Lisboa, Portugal, en noviembre de 1993, puso de ma ni fies to que los sis temas precoordinados han sido preferidos para los ca tálogos en línea de las bi blio tecas generales en numerosos países (Holley, 1993).

Exis ten dos mo da li da des para tra tar es tos lenguajes de indización en la computadora. La primera consiste en mantener estrictamente su característica de precoordinados, registrando la cadena en un solo campo y recuperando por la técnica del truncado y/o mediante una recuperación palabra por palabra; en la segunda los encabezamientos y sus subdivisiones, así como las clases y sus números auxiliares, se re gis tran en dife ren tes subcampos, con lo cual es posible tratarlos con un cierto grado de poscoordinación (Bates, 1988; Buxton, 1990; McIlwaine, 1994).

Por úl ti mo, la po si bi li dad de que el usuario "ex plore los es tan tes" de labiblio te ca desde su propia computadora ha sido suge ri da como una for ma de com ple men tar el ac ce so por ma te ria, ya que una vez que se ha elegido uno o varios documentos me dian te unabús que da con ven cio nal, se pueden revisar las signaturas topográficas y localizar otros documentos que se encuentran adyacentes en el estante porque tra tan so bre la mis ma ma te ria (Klugman, 1989). 


\section{EL CATÁLOGO DE AUTORIDADES}

El control de autoridades es un proceso que consiste en mantener la coherencia entre las formas verbales utilizadas para representar los puntos de acceso al catálogo me dian te laverificación y uni for midad de esas for mas y mos trar las re la ciones entre los nombres (personales, institucionales, geográficos), lostítulosy las materias, mediante una estructura de referencias cruzadas (Taylor, 1992).

El instrumento para llevar a cabo este control es el catálogo de autoridades; para cons truir lo exis ten re glas aplicables a los nombres y títulos, así como reglas aplicables a las materias. Sin embargo, como indica Taylor (1992) desde hace décadas las bibliotecas han abandonado la práctica de controlar los encabezamien tos au tori za dos, tan to para el ca tálogo de fichas como para el catálogo en línea. En el mejor de los casos, los bibliotecarios utilizan como sustituto el mismo catálogo público.

Con el de sa rro llo de los sis te mas en línea se ha manifestadola necesidaddecontar con un ade cua doca tálogo de au to ri da des por los siguientes motivos:

En la etapa de almacenamiento le ofrece al bibliotecario dos ventajas:

1.- Indizar en pantalla sobre la base de los tér mi nos ya au to ri za dos por la biblioteca.

2.- Verificar los tér mi nos y rea li zarcambios globales en algunos o en todos los registros, incluyendo la corrección de errores de tipeado y el reemplazo de términos obsoletos.

A su vez, en la recuperación le ofrece al usuario tres posibilidades:

1.- Recuperar por los términos exactos, ya autorizados, asignados a cada documento.

2.- Navegar en una búsqueda hasta la conclusión deseada, explorando los términos relacionados y los distintos niveles de jerarquía.

3.- Conectar automáticamente los sinóni mos con los tér mi nos de in di za ción, sin necesidad de que el usuario se dé cuenta de esta conexión.

Las ver sio nes en lí nea de los len gua jes de in di za ción fa ci li tan la crea ción de los catálogos de autoridades por materia; inclu so es posible con si de rar los como sustitutos, ya que ofrecen ventajassimilares (Chan, 1990), pero existe en tre ellos una

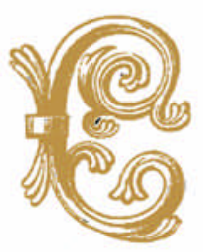

importante diferencia: no todos los términos incluidos en un lenguaje de indiza ción son uti li za dos por una bi blio te ca y a la inversa, todas las bibliotecas tienen la ne ce si dad de crear tér mi nos de in di zación que no se encuentran en el lenguaje deindización, porejem plolosidentificadores de personas, ins ti tu ciones olugares geográficos, así como epígrafes nuevos; es decir, el lenguaje de indización posee tér mi nos que nun ca se uti li za rán en la indización y al mismo tiempo carece de otros que ya se han usado y pueden volverse a ne ce sitar. Por el con tra rio, el ca tálogo de autoridades incluye todos aquellos términos autorizados por la biblioteca, que reflejan su colección con exactitud.

El catálogo de autoridades y el lenguaje de indización en línea se complementan durante la indización, ya que el primero facilita la asignación de términos autorizados y la tarea de verificación, mientras que el segundo es necesario para asignar términos nuevos.
En consecuencia, el catálogo de autorida des y el len gua je de in di za ción en línea se complementan durante la indización, ya que el pri me ro fa ci li ta la asig na ción de términos au to ri za dos y la ta rea de ve ri fica ción, mien tras que el se gun do es ne cesa rio para asig nar tér mi nos nue vos. En la recuperación, el catálogo de autoridades en línea resulta más útil que el lenguaje de in di za ción, por que le ofre ce al usua rio una garantía documental del 100\%.

En los Estados Unidos, la Library of Congress ha desarrollado un módulo de autoridades para el formato MARC en el que se pue den re gis trar las for mas au to rizadas de los encabezamientos y las referencias cruzadas, así como las notas de alcance correspondientes. A su vez, junto con otras bibliotecas cooperantes ha elaborado un catálogo de autoridades para nombres y títulos (LCAF) y otro para materia (LCSAF), disponibles en línea, en microfichas y en CD-ROM.

Aunque el control de autoridades se ha recomendado siempre para los términos ver ba les, la Li brary of Con gress tam bién hadesarrollado otro módulodelformato MARC para el control de las notaciones autorizadas. El módulo fue diseñado entre 1987 y 1988 , en estrecha colaboración con los responsables tanto de LCC como de CDD que son los esquemas de clasificación más difundidos en Estados Uni dos y per mi te re gis trar la no ta ción, la de no mi nación de la cla se, las re fe ren cias cruzadas y las notas de alcance (Guenther, 1992). 


\section{LOS LENGUAJES DE INDIZACIÓN}

\section{C S H}

Duran te mu chotiem polos biblio te carios nor teamericanos han ex presadosuscríti cas y propuestas respecto a LCSH (Bloomfield, 1993; Buckland, 1992; Cochra ne, 1984; Gerhan, 1989; Ho lley y Killheffer, 1982; Klugman, 1989; Reynolds, 1989; Stud well, 1993) e in clut so al gu no se ha per mi ti do su ge rir la eu tanasia para esta lista de encabezamientos (Henige, 1987); las críticas aumentaron significativamente durante el periodo 1970-1979 y más aún en 1980-1989 (Shu bert, 1991), coin ci dien do con la imple men ta ción y eva lua ción de los ca tálogos en línea. Entre los defectos detectados se incluyen la desactualización terminológica, la falta de coherencia y especificidadde los encabezamientos, la carencia de principios teóricos para su cons truc ción, así como su red asis te má tica de referencias cruzadas.

Otro de los requerimientosque se ha hecho a la Library of Congress - aunque sin éxi to has ta el pre sen te- ha sido la redacción de un códi go para los en ca be za mientos de materia, en el que coinciden diversos autores como Chan (1989), Reynolds (1989) y Studwell (1993). Sin embargo, desde 1984 se publica un manual para la asig nación de epígrafes, que ya cuenta con cuatro ediciones (Pietris, 1987; Taylor, 1992).

En 1986 LCSH cam bió la fa mo sa y cen tenaria estructura de referencias cruzadas in ven ta da por Char les A. Cut ter por có digos de tesauro: see (véase) por USE, see also (véase además) por BT y RT, x por UP, xx por NT y RT. Si bien este cambio hasignificado una ayu daporquepermite dis tin guircier tas relaciones je rárquicas y asociativas, LCSH no ha sido convertida en un tesau ro; el es tu dio a fon do de las relaciones entre los encabezamientos queda aún pen dien te y hay que te ner en cuenta que los cambios se hicieron en forma mecanizada (Bates, 1988; Dykstra, 1988; Rolland-Thomas, 1993).

LCSH se publica una vez al año en versión impresa, trimestralmente en microfi chas o enCD-ROM y por se ma na en cinta magnética. También ha sido traducida o adaptada a varios idiomas. En español existe una adaptación que es la Lista de Encabezamientos de Materia para Bi- bliotecas (LEMB), cuya segunda edición fue publicada en 1985 .

\section{C C}

Como es sabido, se trata de un esquema de clasificación universal y jerárquico, pero enu me ra ti vo, no sin té ti co. Estádi vi dido en 45 esquemas individuales, algunos de los cuales tie nen su propioín dice, pero no exis te un ín di ce ge ne ral. El sus tituto obvio son los encabezamientos de LCSH, ya que la mayoría de éstos, aunque no to dos, re mi te a las cla ses de LCC. Distintas experiencias en los Estados Unidos han vinculado ambos lenguajes de in di za ción (Broadbent, 1989; Lar son, 1991; Micco, 1991).

Nancy Williamson ha trabajado en las características y componentes estructurales de LCC para preparar la versión en línea (Chan, 1990), tarea más que difícil por la naturalezaenumerativa de este esquema. El módulo del formato MARC para control de autoridades de los datos de clasifica ción per mi te que las biblio tecas puedan incorporar estas notaciones, pero Guenther (1991) advierte que una versión le gible porcom pu ta doralle garía a te ner cer ca de 500000 cla ses y pre sentar se rios proble mas en cuan to a la ca paci dad de al ma ce na mien to y ve lo ci dad de recuperación.

LCC ha sido tra du ci da al es pa ñol y es utilizada en diver sos países his pa nohablantes.

\section{CDD}

Desde la aparición de la decimoctava edición de 1979 que aumentó las tablas au xi lia res de una a sie te - con virtien do a CDD en una clasificacióncapaz de ordenar la información y no sólo los estantes- y debido a la extensión que alcanzan las notaciones, más de un tercio de las bibliotecas norteamericanas han reclasificadosuscolec cionescon LCC para la signatura topográfica (Chressanthis, 1995; Taylor, 1992). Sin embargo, como ya se mencionó, se han llevado a cabo importan tes ex periencias que han revalorado a CDD como instrumento de almacenamiento y recuperación de información(Drabens tott, 1990; Markey, 1987). En 1988 el oCLC ad qui rió la Fo rest Press y lo gró una ver sión en lí nea con fí nes meramente editoriales. En 1993 se publicó por primera vez Electronic Dewey, que es la ver sión en CD-ROM de CDD para in- dización y recuperación; está basada en la vigésima edición y cuenta con nueve índices (Tro tter, 1995). Por otra par te, el formato MARC incluye tanto la notación de CDD asignada por la Library of Congress como la no ta ción asig na da por cada biblioteca individual. Además, el módulo del formato MARC para el control de autoridades de los datos de clasificación per mi te que cadabiblio te ca pue da in corporar sus notaciones autorizadas (Guenther, 1991).

La terce ray úl ti ma edi ción de CDD en espa ñol ha sido pu bli ca da en 1995 y se basa en la vigésima edición en inglés.

\section{CDU}

En 1993 la FID anunció oficialmente la aparición de la versión de CDU legible por computadora, basada en el Master Re fe ren ce File, base de da tos del Con sorcio CDU que cuenta con 60.000 clases. Diversas experiencias internacionales han demostrado la utilidad de CDU para el almacenamiento y recuperación de la informaciónensistemascomputarizados (Buxton, 1990; McIlwaine, 1994; Strachan y Oomes, 1995).

La última edición abreviada española es la sex ta de 1991. La Aso cia ción Es pa ñola de Nor ma li za ción (AENOR), que es el organismo responsable de CDU en nuestra lengua, ha publicado ya una versión informática de esta sexta edición abreviada y espera publicar la primera edición media en español a fines de 1995.

\section{Otros lenguajes de indización}

Los principales avances en relación con los lenguajes de indización incluyen los tesauros, las clasificaciones facetadas, PRECIS y la con ci lia ción de dis tin tos lenguajes.

El tesauro es un lenguaje de indización cuyos términos conforman un vocabulario controlado y se estructuran en un sistema de relaciones equivalentes, jerárquicas y asociativas, de manera que un mismo lenguaje proporciona el acceso al fa béti co y el ac ce so sis te mático. Nancy Williamson (1989a) ha señalado que los diseñadores de sistemas de información hanig no ra do las ven tajas del com ponente sistemático del tesauro, que puede ser de gran uti li dad para el usua rio du ran te la recuperación, mediante la exhibición de las relaciones jerárquicas y asociativas. Dado su carácter poscoordinado, el 
tesau ro se adap ta me jor al uso de los operadores boolea nos en la com pu ta do ra y la indización resulta menos costosa. Desde su apa ri ción en la dé ca da de los cin cuen ta se ha registrado una proliferación de tesauros especializados, pero desafortuna damen te noexis te un tesau ro universal para las bibliotecas generales.

En cuanto a las clasificaciones facetadas se ha demostrado su eficiencia en sistemas pos co or di nados, en la me didaen que las cla ses pro du ci das por el análi sis facetal estén representadas por una notación tal que permita poscoordinarla con los operadores booleanos, al mismo tiempo que posibilite la exploración jerárquica por medio del truncado (Gödert, 1991). En 1977 se comenzó a publicar en Gran Bre ta ña la se gun da edi ción de la Bliss BibliographicClassification (B BC), bajo la di rec ción de Jack Mills; como es sa bi do, se tra ta de un es que ma uni ver sal y face tado.

La ma yoría de los es que mas in di viduales ya han sido publicados, pero el esquema completo se encuentra aún en curso de publicación (Foskett, 1982; Taylor, 1992; Thomas, 1995). Otra clasificación uni ver sal y face ta da es la Co lon Clas si fication ( $\mathrm{CC}$ ), cuya sép ti ma edi ción fue pur blicada en 1988 por la Sarada Ranganathan Endowment; a pesar de sus sesenta años de existencia, este esquema no ha logrado imponerse en las bibliotecas generales ni competir en difusión y uso con las tradicionales CDD y CDU. Ni BBC ni CC cuentan con versiones legibles por computadora, ni han sido traducidas al español.

Por su par te PRECIS es un sis te ma crea do en Gran Bretaña por Derek Austin, que entró en servicio en la British National Bibliography en 1971. Más que un lenguaje de indización se trata de un procedimiento basado en el conceptode vocabulario open-ended, es decir que los términos pueden ser admitidos en cualquier mo men to en que apa rez can en la literatura.

Una vez que el documento ha sido analizado, los con cep tos son or de na dos como un tí tu lo en len gua na tu ral, for man do una ca de na de tér mi nos, cada uno de los cuales está pre ce di do por un ope ra dor de terminado. Aque llos tér mi nos que apa re cen en la posición líder son marcados y las estructuras semánticas se añaden en forma de referencias de véase además para cualquiera de los términos de la cadena. También se incluyen los códigos de manipulación para la computadora y ésta se encarga del ordenamiento (Dykstra, 1989; Lancaster, 1991; Rowley, 1988). Lamentablemente, PRECIS resul ta cos toso de bi do al tiem po que con su me el análi sis sin tác ti co y la co di fi ca ción de las cadenas; a fines de 1990 la British Library se vio for za da a sus ti tuir lo por un sis te ma menos sofisticado denominado COMPASS (Holley, 1993; Taylor, 1992); por el mismo motivo la Library of Congress nunca adoptó PRECIS, a pesar de ciertas presiones de la comunidad bibliotecaria norteamericana (Holley y Killheffer, 1982).

\section{Conciliación de lenguajes de indización}

Con la pro li fe ración de tesau ros y cla sificaciones es pecializados, sehaproducido un au men to en la in com pa tibilidad de los ca tá lo gos en lí nea, tan to en el ni vel de especificidad, como en la exhaustividad, los términos compuestos, los sinónimos y las relaciones entre los términos. La compatibilización o conciliación de estos lenguajes incluye las técnicas de conexión y fusión, así como los macrotesauros (Aitchison y Gilchrist, 1987; Niehoff y Mack, 1985; Rada, 1987; Taylor, 1992).

Ejemplos de esta con ci lia ción son el sistema de conexión de vocabulario desarrollado por Battelle Columbus Laboratories de Estados Unidos (Niehoff y Mack, 1985); el sis te ma uni fi ca do de lenguajes médicos de la U.S. National Library of Medicine (Squires, 1993), o el sistema operacional de la NASA que conecta sus propios términos deindización con aqué llos del U.S. De part ment of Defen se, me dian te una tabla de tra duc ción o léxico y un programa que determina las reglas a seguir (Silvester y Klingbiel, 1993).

Douglas J. Foskett (1991) ha manifestado que B BC puede ser el mejor lenguaje de conciliación. Este esquema facetado tiene una doble función: a) dado que sus clases principales han sido desarrolladas en es que mas in di vi dua les con un alto nivelde es pecificidad, es posiblein dizaren profundidad aún la información más específica, y b) todos los esquemasindividuales forman un solo esquema universal, totalmente compatible, porque al

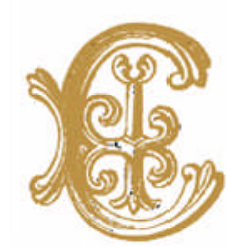

Con la proliferación de tesauros y clasificaciones especializados, se ha producido un aumento en la incompatibilidad de los catálogos en línea 
ha ber sido ela bo ra do con la mis ma me todología de análisis facetal, estructurado con las mismas reglas y dotado de un mismo sistema de notación, garantiza una homogeneidad global.

\section{CONCLUSIONES}

ComoseñalaMandel(1991), lanaturaleza inexacta y subjetiva de la búsqueda por materia requiere múltiples rutas de accesoparafacilitarlacoincidenciaen tre los tér mi nos del usua rio y el vo ca bu la rio del sistema. Por su parte, Chan (1990) y Lar son (1991b) es tán de acuer do con que no basta incluir más términos, sino en combinar distintos tipos de términos ya sean pa la bras cla ves de la len gua na tu ral, asícomo tér mi nos con tro la dos de ac ce so verbal (encabezamientos de materia o descriptores) y de acceso sistemático (notaciones).

Seresu men a con ti nua ción las di ferentes propuestas para mejorar la indización por ma te ria de las mo no gra fías en los catálogos en línea de bibliotecas generales (Larson, 1991a):

1.- Mayor can ti dad de tér mi nos verbales controlados (encabezamientos de materia o descriptores): asignar más términosverbalescontrolados por registro y explotar las versiones en línea de los catálogos de autoridades o los lenguajes de indización para proporcionar mayor coherencia a los indizadores y orientación a los usuarios.

2.- Mayor cantidad de palabras claves (lengua natural): explotar los términos individuales de cada campo por medio de palabras claves, añadir pala bras cla ves de tí tu loy/ore su men en cada registro y, en el caso de las monografías, incluir palabras claves de sumarios e índices analíticos.

3.- Mayor cantidad de notaciones: asignar notaciones a todos los registros y añadir los términos verbales derivados de los esquemas de clasificación y sus índices.

Por otro lado, si bien los lenguajes de indización tradicionales ( $\mathrm{LCSH}$, LCC, CDD, CDU) han sido muy criticados por su carácter precoordinado, su dificultad para procesarlos en la computadora, la obsolescencia de su terminología, su orde na mien to por dis ci pli nas y no por conceptos, etcétera, cuentan con una amplia difusión internacional, han sido traducidos a va rios idio mas y ad mi nis tra dos durante décadas por organizaciones responsables (Library of Congress, Forest Press, FID), que han garantizado su continuidad.

Las dificultades eco nó mi cas de este úl timo cuarto de siglo, que en mayor o menor grado afectan a las bibliotecas de todo el mun do, im pi den o ha cen muy difícil que puedan crearse nuevos lenguajes de indización universales más eficientes o modificar sustancialmente los tra di cio na les y, más aún, po ner en prác ticauna posible recla si fica ción o re in di za ción de los mi les de mi llo nes de re gis tros bibliográficos existentes (Chan, 1990; Holley, 1989; Mandel, 1991).

De ma ne ra que por el mo men to no se vislum bran cam bios en el uso de los len guajes de in di za ción para los ca tá lo gos en línea de las bibliotecas generales, sino un uso más efi cien te de los ac tua les me diante el au men to de los pun tos de ac ce so por materia en cada registro y su comple- mento con la lengua natural (Williamson, 1989b).

En Argentina - y tal vez suceda lo mismoen otros países la ti no ame rica nos-la mayoría de los catálogos informatizados de las bibliotecas generales no cuenta aún con un acceso remoto que facilite la consulta al usuario; en consecuencia, no se pue de co nocer con exac ti tud el de sempe ño de los usua rios du ran te la bús que da por materia.

Sinembargo, los resultados preliminares de una evaluación de estos sistemas en nuestro medio (Pichinini y Martínez, 1995) demuestran que los puntos de acceso por materia son prácticamente los mismos que en la ficha: uno o dos encabezamientos y un número clasificatorio; excepcionalmente palabras claves de títu lo y en nin gún caso el re su men. De esta for ma, es posible an ti ci par un alto ín di ce de fracasos en la búsqueda por materia, tal como se ha podido detectar en otros países (Larson, 1991b; Su, 1994).

Las propuestas del formato aumentado que se han mencionado anteriormente, en especial la inclusión de un resumen, im pli can un au men to en los cos tos, tan to en la capacidad de almacenamiento y procesamiento de datos, como en recursos hu ma nos ca lifi ca dos, que qui zás muchas bibliotecas no es tén en con diciones de afrontar. Por otra parte, los lenguajes documentales universales en español (LEMB, LCC , CDD , CDU) demoran mucho tiem poen ac tua li zar se y, has ta don de se sabe, sólo CDU está dis po ni ble en versión informática en nuestro idioma, con lo cual las di fi cul ta des para la in di za ción son aún mayores.

Quizás las experiencias obtenidas en otros países, tal como se ha intentado pre sen tar en esta re vi sión, pue dan con tribuir a una am plia dis cu sión de es tos problemas, a la prevención de los mismos errores y al diseño de catálogos en línea más efi cien tes para los usua rios de nuestras bibliotecas.

\section{BIBLIOGRAFÍA}

Aitchison J, Gilchrist A. Thesaurusconstruction. 2 ed. London: Aslib, 1987.

Bates M. "How to use con trolled vo ca bu la ries more effec ti vely in on li ne sear ching". Online 1988; 12(6):45-56.

Bates M. "Rethin king sub ject ca ta loging in the on li ne en vi ron ment". Li brary re sources and technical services 1989; 33(4):400-12. 
Bloomfield M. “A look at subject headings: a plea for standardization”. Cataloging and classification quarterly 1993; 16(1):119-24.

Broadbent E. "The online catalog: dictionary, classified, or both?" Cataloging and classification quarterly 1989; 10(2):105-17.

Buck land MK. "Agen da for on li ne ca ta log de sig ners."Information te chnologyandli braries $1992 ; 11(2): 157-62$.

Buxton AB. "Computer searching of UDC numbers." Journal of documentation $1990 ; 46(3): 193-127$.

Carlyle A. "Matching LCSH and user vocabulary in the librarycatalog." Cataloging and classification quarterly 1989;10(2):37-63.

Chan LM. "A subject cataloging code?" Cataloging and classification quarterly $1989 ; 10(2): 199-202$.

- _ . "Subject analysis tools online: the challenge ahead." Information technology and libraries 1990; 9(3):258-62.

Chres santhis JD. "The re clas si fi ca tion de ci sion: Dewey or Li brary of Con gress." $C a$ taloging and clas sification quarterly 1995; 19(3/4):169-82.

Cochrane PA. "Modern subject access in the online age." American libraries 1984; $15(2): 80-3 ; 15(3): 145-8,150 ; 15(4): 250-2,254-5 ; 15(5): 336-9 ; 15(6): 438-41$; $15(7): 527-9$

Cousins SA. "Enhancing sub ject ac cess to OPACS: controlledvocabulary vs. natural language." Journalofdocumentation 1992; 48(3):291-309.

Cul kin PB. "Rethin king OPACS: the de sign of as ser ti ve in for ma tion sys tems." Information technology and libraries 1989; 8(2):172-7.

Drabenstott KM, y otros. "Analysis of a bibliographic database enhanced with a library classification." Library resources and technical services 1990 ; 34(2): 179-98.

Dubois CPR. "Free text vs. controlled vocabulary: a reassessment." Online review $1987 ; 11(4): 131-6$.

Dykstra M. "LC subject headings disguised as a thesaurus." Library journal 1988; $113: 42-6$

__. "PRECIS in the on li ne ca ta log." Catalogingandclas sification quarterly $1989 ;$ 10(2): 81-94.

Foskett D. "Concerning general and specialclassification." Internationalclassification $1991 ; 18(2): 87-91$.

Foskett AC. The subjectapproachto information. 4 ed. Lon don: Cli ve Bin gley, 1982.

Gerhan DR. "LCSH in vivo: sub ject sear ching per for man ce and stra tegy in the OPAC era." Journal of academiclibrarianship 1989; 15(2):83-9.

Gödert W. "Facetclas si fication in on li ne re trie val."Internationalclassification 1991; 18(2):98-109.

Guenther RS. "The de ve lopment and im ple men ta tion of theUSMARC for mat for classification data." Information technology and libraries 1992; 11(2):120-31.

He ni ge D. "Li brary of Con gress subject hea dings: is eutha na sia the ans wer?" Cataloging and classification quarterly 1987; 8(1):7-19 y 21-3.

Holley RP, Killhe ffer RE. "Is the re an ans wer to the sub ject ac cess cri sis?" Cataloging and classification quarterly 1982;1(2/3):125-33.

- - "Sub ject ac cess in the on li ne ca ta log." Catalogingandclassificationquarterly $1989 ; 10(2): 3-8$.

Holley RP. "Report on the IFLA Satellite Meeting Subject Indexing: Principles and Practices in the 90's, August 17-18, 1993, Lisbon, Portugal." Cataloging and classification quarterly 1993; 18(2):87-100.

Kern-SimirenkoC. "OPAC user logs: implications for bibliographic ins truction." $L i$ brary hi tech 1983; 1(3):27-35.

Klugman S. "Failures in subject retrieval." Cataloging and classification quarterly $1989 ; 10(2): 9-35$.

Knutson G. "Sub ject enhan ce ment: re port on an ex pe ri ment." College and research libraries 1991; 52(1):65-9.

Lancaster FW. Indexing andabstracting. Cham paign: Uni ver sity of Illi nois, 1991. 
Lar son RR. "Clas sification clustering, probabilis tic in for mation re trieval and theonlinecatalog." Libraryquarterly 1991a; 61(2):133-73.

Lar son RR. "The de cli ne of sub ject sear ching: long-term trends and pat terns of in dex use in an online catalog." Journal of the American Society for Information Science 1991b; 42(4):197-215.

McIlwai ne IC, Bux ton A. Guía para uso de la CDU: Clasificacióndecimaluniversal. Madrid: AENOR, 1994.

Mandel CA. "Cataloging for access." Referencelibrarian 1991; (34):61-8.

Markey K. "Sear ching and browsing the Dewey De ci mal Clas si fi ca tion in an on li ne catalog." Cataloging and classificationquarterly 1987; 7(3):37-68.

Micco M. "The next ge ne ra tion of on li ne pu blic ac cess ca ta logs: a new look at sub ject access using hypermedia." Cataloging and classfication quarterly 1991; 13(4):103-29.

Niehoff R, Mack G. "The vocabu lary swit ching sys tem: des crip tion of evalua tion studies." International classification 1985; 12(1):2-6.

Pe ters TA. "When smart peop le fail: an analysis of the tran sac tion log of an on li ne public accesscatalog." Journal of acade mic librarianship 1989; 15(5):267-73.

Pi chi ni ni M, Martínez AM. Catálogos en línea de bibliote cas bo nae ren ses: re sulta dos preliminares de una encuesta [Resumen]. Curso de Actualización sobre Acceso en Línea a los Catálogos de Bibliotecas. La Plata: UNLP, 1995.

Pie tris MKD. "LC: new ma nual, not code, ne e ded."Americanlibraries 1987; 18:958.

Rada R. "Con nec ting and eva lua ting thesau ri: issues and ca ses." Internationalclassification 1987; 14(2):63-9.

Reynolds SJ. "In theory the re is no solu tion: the im pe di ments to a sub ject ca ta lo ging code." Library quarterly 1989; 59(3):223-38.

Ro lland- Tho mas P. "Thesau ral co des: an apprai sal of their use in the Li brary of Congress subject headings." Cataloging and classification quarterly 1993; $16(2): 71-91$

Rowley JE. Abstracting andindexing. 2 ed. Lon don: Cli ve Bin gley, 1988.

Settel B, Cochrane PA. "Augmenting subject descriptions for books in online catalogs." Database 1982; 5(4):29-37.

Shubert SB. "Cri ti cal views of LCSH ten years la ter: abibliographic as say." Cataloging and classification quarterly, 1992; 15:37-97.

Silvester JP, Klingbiel PH. "An operational system for subject switching between controlled vocabularies." Information processing and management 1993; 29(1):47-59.

Squires SJ. "Ac cess to bio me dical in for mation: the uni fed me dical lan gua gesys tem." Library trends 1993; 42(1):127-51.

Strachan PD, Oomes FMH. "Universal decimal classification update." Cataloging and classification quarterly 1995; 19(3/4):119-32.

Studwell WE. "LC's head in the sand, or, why the subject cataloging manual is not enough." Technical services quarterly 1993; 10(3):45-50.

Su SF. "Dia lo gue with anOPAC: how vi sio nary was Swan son in 1964?"Li brary quarterly 1994; 64(2):130-61.

SvenoniusE. "Unans we red questions in the de sign of con trolled vo cabu la ries."Journal of the American Society of Information Science 1986; 37(5):331-40.

Taylor A. Bohdan S. Wynar: Introduction tocataloging andclassification. 8 ed. Englewood: Libraries Un li mi ted, 1992.

Thomas AR. "Bliss classification update." Cataloging and classification quarterly $1995 ; 19(3 / 4): 105-18$.

Trotter R. "Elec tro nic Dewey: the CD-ROMver sion of the Dewey De ci malClas si fication." Cataloging and classificationquarterly 1995; 19(3/4):213-34.

Williamson NJ. "The role of clas si fi ca tion in on li ne sys tems." Ca ta lo ging and clas sification quarterly 1989a; 10(1):95-104.

- - "Classification in the computer age." International classification 1989b; $16(1): 30-4$. 